\title{
Child Welfare Workers' Home Visit Risks and Safety Experiences in the USA: A Qualitative Approach
}

\author{
HaeJung Kim ${ }^{1, *}$, Karen M. Hopkins ${ }^{2}$ \\ ${ }^{1}$ School of Social Work, Eberly College of Arts and Sciences, West Virginia University, USA \\ ${ }^{2}$ School of Social Work, University of Maryland, USA
}

\begin{abstract}
Human service providers, including child welfare workers, who routinely visit clients' homes frequently face unsafe working conditions during their home visits. Although previous studies have emphasized the importance of safety issues in helping professionals, not many studies have specifically examined home visiting concerns. The purpose of this study is to explore individual, situational, and environmental characteristics that increase workers' home visit risks. Qualitative interviews were conducted with nine child welfare workers that completed a survey as part of a larger study on retention. From qualitative interviews, child welfare workers commonly experienced verbal or physical threats initiated by clients or community members. Situational and community factors were also identified with increased risk in home visits. For many, evening home visits to see children's parents usually increased the level of risk. Visiting communities with a violent reputation, receiving little support from police or community members, and a lack of community resources in risky situations posed increased risks during home visits. Overall, the current study may provide a better understanding and incentive for the further development of safety training in educational curriculums and agencies, and legislation and state guidelines that improve the safety of helping professionals.
\end{abstract}

Keywords Workplace Violence, Child Welfare, Home Visits Risks, Safety Experiences

\section{Introduction}

The social work profession has been actively involved in child welfare to help prevent child maltreatment and enhance child safety and well-being for over 100 years (NASW, 2013). Child welfare workers are on the front lines providing an array of prevention and intervention services to help children and families. Among the programs and services offered by child welfare workers, in-home services play an important role in assisting families with concerns related to child abuse and neglect (Child Welfare Information Gateway, 2014). Since some studies show that removing children from their families can have negative effects on children's psychological and emotional health (Doyle, 2007; Kolko et al., 2010), in-home services have been particularly emphasized in child welfare practice.

Child welfare workers spend the majority of their time visiting clients' homes in order to assess ongoing service needs of children and families and monitor children's safety and well-being. Although home visiting is distinguished from in-home services, studies have indicated that home visiting could help to reduce child maltreatment and improve parent-child attachment relationships and child development outcomes (Duggan et al., 2007; Staerkel and Spieker, 2006). However, home visits are also critical risk factors for "workplace" violence. Human service professionals, including child welfare workers, who routinely visit clients' homes frequently face unsafe working conditions during their home visits (Shields and Kiser, 2003).

Children and youth service workers have been shown to be the most vulnerable to clients' violence. The American Federation of State, County, and Municipal Employees (2011) reported that about 70 percent of child welfare workers in the United States have been victims of violence or threats while they work. According to this study, 90 percent of former child welfare workers reported that they experienced some types of verbal threats, 30 percent experienced physical attacks, and 13 percent had been threatened with weapons. Child welfare workers also experience high levels of secondary traumatic stress and burnout compared to other professions (Sprang et al., 2011), and an experience with violence heavily influences negative personal and organizational outcomes in the short-term and long term (Enosh et al., 2013; Kim \& Hopkins, 2015). Therefore, understanding the issues, reactions, and responses associated with this type of workplace violence and developing intervention strategies are critical to the 
well-being of child welfare workers in their jobs and to the optimal effectiveness of agencies.

\section{Individual, Situational, and Environmental Characteristics for Home Visit Risks}

Applying the Cognitive-Perceptual Model of Risk in Home Visiting (CPMRHV) developed by Kendra and George (2001) in the nursing profession, the level of home visiting risks are influenced not only by workers' individual characteristics and circumstances, but also by the situational and environmental context of the home visits. Specifically, Kendra and George (2001) identified workers' attributes that can influence cognition and perception of risk; these attributes include age, gender, personal motivation, education level, degree of autonomy on the job, years of practice, and years of experience with home visiting, workers' self-concept, life experiences, income, availability of support system, and personal and family stressors. Based on this theoretical framework, workers' perception of home visit risks is also dependent on situational and environmental factors related to the agency. For example, interpersonal relationships between worker and supervisor, organizational policies and strategies for personal safety, and organizational structure also impact workers' perception of home visit risks. In addition, environmental factors, such as population density in the area of the visits, racial and economic instability, and lack of social resources (i.e., police), influence workers' perception of uncertainty or ambivalence about home visiting, which can ultimately increase their level of home visit risks.

Although a limited number of studies have addressed home visit risks in the social work profession, this framework is generally supported by previous empirical studies. Some researchers (Astor, Behre, Wallace, \& Fravil, 1998; Balloch, Pahl, \& McLean, 1998; Briggs, Broadhurst, \& Hawkins, 2004; Jayaratne et al., 2004; Koritas, Coles, \& Boyle, 2010) have supported CPMRHV model in that there are differences in the number of violent incidents, depending on workers' age, gender, ethnicity, and tenure. For example, studies showed that male or younger social workers were more likely to be targets of clients' violence than female or older workers (Balloch et al., 1998; Newhill, 1996). Jayaratne and colleagues (2004) found that male social workers in the public sector were more likely to report physical threats than females, but not verbal abuse. Additionally, younger workers $(<45$ years) reported more incidents of physical threats, verbal abuse, and sexual harassment than older workers $(\geq 45)$. In terms of race, studies (Jayaratne et al., 2004; Astor at al., 1998) found significant differences between African American workers and other racial groups, indicating that African American workers in the public sector experienced less fear of future violence than other groups. However, as researchers identified, this finding was confounded by community characteristics; whether they worked in an urban or rural setting. For example, in the study of Astor and colleagues, most social workers of color had worked in urban areas. Therefore, when controlling for community characteristics, no differences might be found between African American and Caucasian social workers.

Although only a few empirical studies have focused on situational/environmental factors that increased home visit risks, available literature consistently showed that workers in dangerous communities are likely to experience a higher level of risk than workers in less dangerous communities. For example, most research related to workplace violence in the social work field has focused on differences in the amount of client violence between rural and urban communities. Workers in urban agencies were more likely to experience threats of violence from clients or fear for their personal safety during their home visits compared to those in rural agencies (Astor et al, 1998; Jayaratne et al. 2004; Shields and Kiser, 2003). Particularly for home visits, Spencer and Munch (2003) identified several main risk factors for workers during outreach interventions: worker vehicles, the community at large, access to neighborhood exit, and clients' residences. For instance, the external appearance of clients' residences, including improper general maintenance and lightning, the presence of individuals other than clients in the home, and observable illegal paraphernalia can also be threatening for workers during their home visits.

In sum, although previous studies have emphasized the importance of safety issues, there is limited research that specifically examines child welfare workers' home visiting concerns. Little is known about the environmental or situational context that child welfare workers face during their routine home visits. Therefore, the purpose of this qualitative study is to explore child welfare workers' safety experiences during home visits. Specific attention is given to exploring individual, situational, and environmental characteristics that increase workers' home visit risks. The study is guided by two research questions:

Research question 1. What are the "workplace" violence perceptions and experiences of child welfare workers?

Research Question 2. What individual, situational, and environmental risks do child welfare workers face during home visits?

\section{Methods}

\section{Study Design and Sample}

This study was part of a larger mixed-methods study about child welfare workers' retention (Author, 2012). Initial purpose of the larger study was to examine multiple factors that may predict perception of risk and its consequences on child welfare workers' personal and organizational outcomes. The three objectives of the larger study were to: understand child welfare workers' workplace violence experiences; examine predictors of safety concerns; and investigate a relationship between safety concerns and 
job withdrawal. Therefore, a mixed-methods sequential explanatory design that purposefully selects participants for a follow-up, in-depth, qualitative study (Creswell and Clark, 2007) was employed. This design allows researchers to conduct additional qualitative interviews in order to explain why certain individual and organizational factors, tested in the quantitative first phase, are significant or not significant predictors of child welfare workers' job withdrawal.

The specific aim of the present study was a qualitative in-depth exploration of child welfare workers' home visit risks and a purposeful intensity sampling was used for this study. From those workers who completed a quantitative survey in 2007, a small number of interview participants were later selected for follow-up. As the sample size for the quantitative surveys was 600 child welfare workers, the pool for potential qualitative interview participants was narrowed using two criteria; safety concerns scores and job withdrawal scores. With the use of $30 \%$ cut points, 58 manageable potential participants were identified. A group of 58 child welfare workers who scored high on safety concerns (upper $30 \%$ ) and scored high on intention for job withdrawal (upper $30 \%$ ) were selected as potential participants. Among them, 21 child welfare workers had left the agency during 2007 2011, which yielded 37 potential participants for the study.

First, invitation letters were sent to the 37 potential participants via emails, which included the purpose of the study, how the respondent was chosen (emphasizing that this was a follow-up study of the original project), and possible date, time, and duration of the interview. The email also included an assurance of confidentiality, emphasizing that no one at their agency would have access to transcripts or interview tapes. A week after the email, the researcher mailed the invitation letters to them at their agency address, and the employees were asked to confirm their participation for the interview. Two weeks after sending the initial letter, the researcher sent a reminder, which encouraged employees' participation in the individual interview. Sixteen workers responded to invitation letters. Among the sixteen workers, four declined the interview and three workers failed to contact the researcher to follow up with scheduling interviews. Therefore, nine workers agreed to participate in the interviews.

\section{Interview Procedures}

Data were collected from March to May, 2011. Interviews took place at a participant-preferred location (e.g., classroom at the university, meeting room at a local public library, and participants' homes), lasting from 1 hour to 1-1/2 hours. All participants were interviewed one time. The study was approved by the University Institutional Review Board (IRB-00047093). Twenty dollars was given to each respondent who participated in an interview to compensate for their time and efforts. Also, additional parking fees were provided to participants who traveled to the University for the interview. A semi-structured interview guide was developed as the primary method of data collection. Participants were asked a series of questions about types of workplace violence behaviors and events that they experienced during home visits, and their perceptions of risk related to worker characteristics, situational characteristics, and community characteristics. For example, sample questions included "Could you please tell me about your safety experiences during home visits?", "When do you feel safety concerns?", "What have you done when you experienced safety concerns?", "How have your safety experiences influenced your personal and organizational outcomes (e.g., stress, commitment, and intention to leave)?, "How have coworkers or supervisors responded when you have expressed safety concerns?"

\section{Qualitative Data Analysis}

All interviews were audio-taped and transcribed verbatim with permission from participants. Interviews were coded as soon as transcripts become available. A thematic analysis (Boyatzis, 1998), which involves searching through data to identify any recurrent patterns, was used for qualitative data. QSR NVivo 10.0 was used for the analysis of the collected data from nine child welfare workers.

As a first step, the researcher began the analysis through open coding by reading each transcript word by word and line by line and naming the concepts that emerged from the data. All codes were then analyzed and similar codes or concepts were combined or collapsed into categories, while seeking to answer research questions. At this stage, most codes were developed on earlier research from the CPMRHV model developed by Kendra and George (2001) and a home visit risk assessment tool by Spencer and Munch (2003). To establish and enhance the trustworthiness of findings, triangulation, peer debriefing, and member checking strategies were employed. First, from the data collection to data analysis, the researcher used triangulation, particularly triangulation by data source strategy to reduce the threat to trustworthiness. In addition to qualitative in-depth interviews, the data was gathered from the quantitative online survey and field notes from the researcher's observations during the interview. Second, peer debriefing was employed to counter the researcher's bias. During the course of the study, this researcher met with an expert qualitative researcher with an extensive knowledge of this study on a regular schedule and discussed the issues regarding data collection and analysis. This researcher obtained feedback and support through debriefing; this reduced threats of possible bias and ultimately contributed to the rigor of a qualitative approach. Third, for member checking to verify the accuracy and validity of the data, upon completion of data analysis, summarized results were returned to participants to review the interview summaries and findings. 
Table 1. Demographic Characteristics for the Qualitative Interview Participants

\begin{tabular}{|c|c|c|c|c|c|c|}
\hline & Age & Tenure in years & Position & Education & Gender & Race \\
\hline Amy & 53 & 20 & Line Worker & MSW & Female & African American \\
\hline Michelle & 43 & 10 & Line Worker & MSW & Female & African American \\
\hline Jack & 39 & 14 & Supervisor & MSW & Male & Caucasian \\
\hline Angela & 39 & 16 & Line Worker & MSW & Female & African American \\
\hline Nicole & 40 & 10 & Line Worker & Non-MSW & Female & African American \\
\hline Jay & 60 & 25 & Line Worker & MSW & Female & African American \\
\hline Tisha & 50 & 12 & Line Worker & Non-MSW & Female & African American \\
\hline Pat & 47 & 17 & Supervisor & MSW & Female & Caucasian \\
\hline Melissa & 36 & 6 & Line Worker & MSW & Female & African American \\
\hline
\end{tabular}

\section{Findings}

\section{Demographic Characteristics}

Demographic characteristics for the qualitative interview participants are presented in Table 1. Most interview participants were female, African American, and line workers who hold a Master of Social Work (MSW) degree, and who were working in urban communities at the time of the interview. Two interview participants were currently in supervisory positions and had been promoted since 2007 . Participants' average age was 45.2 years with an average of 14.4 years of experience in the social work field. Based on the survey data collected in 2007 , these participants were older, perceived less support from supervisors and the organization, and they were less committed to their career in the child welfare field than the rest of the survey respondents. These respondents also perceived less respect from communities and more negative public perception about their agency compared to the total sample.

\section{Workplace Violence Experiences}

Based on the qualitative interviews, verbal or physical threats initiated by clients or community members was the most common form of violence. For example, one of the interview participants, Michelle, said that she frequently experienced yelling, swearing, and verbal insults directed toward her by clients or their family members. Interview participants with current or past experiences in child protective services were particularly exposed to verbally abusive situations while working with involuntary parents. Child protective services workers sometimes are involved in removing children from their biological parents, and the parents can be oppositional, resistant, or angry toward the workers. One interview participant, Amy, said "the most challenging part for a child welfare worker is probably the process of dealing with the child's separation from the parents." Similarly, Pat, a current in-home family services supervisor, recalled her previous experiences in a family preservation unit where she had experienced verbal threats of harm by involuntary parents:

The case was passed along to us to provide the continuing in-home services. He knew that there is a possibility that if he did not get himself into (drug abuse) treatment and improve his parenting, there is possibility that his children could be removed from his care. I often had to remove children from a parent, but this man said "if you remove my children from me, I will kill you. (Pat)

Another theme that emerged from the qualitative interviews was unsafe environmental conditions in clients' residences. In home visiting situations, the child welfare workers felt uneasiness, concerns, or fear due to the presence of animals, presence of unexpected people in client's homes, and sanitation problems. For example, Pat recalled one of the most pest infested houses that she had ever seen and she was worried about her personal health as well as her family's health conditions:

I could see on the table a moving mass of bugs crawl all over and they were on the wall, they were in the curtains, they were on the ceilings. There were droppings so we would have to be aware of what might have been dropping on us. There were mice that were in all of the cabinets and they would be like coming out and running across the top of the cabinets and it was the most pest infected house that I had ever seen.... walk into a home where bed bugs are present and that is definitely on the rise. It means that the workers can bring home bed bugs to their own home. That is the safety issue. There are (my) other family members to [be affected by the] infestation. (Pat)

This suggested that sometimes child welfare workers were more concerned about their own health conditions rather than being a victim of violence while they were visiting a home with extreme sanitary challenges.

\section{Home Visit Risks}

\section{Child Welfare Worker Characteristics: Age, Experience, Gender, and Race}

Most interview participants believed that certain demographic characteristics (i.e., age, race, gender, tenure) are associated with child welfare workers' perceptions of risk. They pointed out that age and tenure, in particular, 
were critical factors related to their perception of risk. Several of the interview participants reported that young child welfare workers just finishing graduate school expressed more safety concerns during home visits. Michelle noted that young child welfare workers have a lack of skills, knowledge, and experiences in dealing with violent situations; therefore, they are more likely to be victims of violence compared to experienced child welfare workers. She also noted that some young child welfare workers are sometimes too judgmental toward clients' life situations, with an attitude of "I know everything; I've learned it all from the master's program" and it made it harder for them to deal with violent situations.

Angela noted that the main difference between the groups that perceive a high level of risk and a low level of risk is work experience, pointing out that experienced workers not only possess strong clinical skills or knowledge in dealing with violent situations, but they also have enough information about community resources that they can use when they are exposed to violent situations.

They [experienced workers] know the area. We have new workers that are not familiar with the area that they work in and not knowing surroundings. It can create a certain level of anxiety. Learn the culture of that community that we are working in, that [learn the culture] sometimes is new to a new worker.... Also knowing resources, what is available to workers in terms of their safety when they are working with families is different from a veteran worker and from a new worker. (Angela)

Melissa agreed that experience in child welfare is one of the factors that is associated with the level of perceived risk. However, different from other interview participants, she noted that experienced child welfare workers could more easily receive support from other colleagues in visiting clients' homes, which can lead to fewer safety concerns during home visits among experienced child welfare workers.

So the very first time (when) I was hired as a DSS social worker, in that time I had no bond with a colleague. But, I am kind of getting accustomed to the (organizational) culture, much more comfortable to work in child welfare, and then (I) built up (relationship) with colleagues, so then I can much easier ask other people for help with the home visit. (Melissa)

A few interview participants stated that race and gender were related to the perceived level of risk. Tisha noted that race issues were associated more often with concerns for "black women going into white neighborhoods," or "white women going into black or Hispanic neighborhoods." Michelle also stated that it was more threatening for staff of a particular race to go into an area where the majority of people were of a different race. Similarly, gender issues were frequently associated with concerns for "female workers meeting male clients." For example, Michelle said that she was not feeling comfortable visiting a male client by herself, so she frequently asked a male co-worker to go with her if she needed to see male clients:

When I first started working there [DSS], I had a coworker, he was a male, I would always ask him to go with me to certain visits. If I meet a male client, I will ask him [coworker] to come with me because I don't feel comfortable as a woman going into this home with this male client by myself. (Michelle)

\section{Situational Characteristics: Evening Visits and Home Visits Alone}

One of the major themes that led participants to feel fear or concern was making frequent evening home visits by themselves. For most, evening home visits to see children's parents for a required assessment usually increases the level of risk. One participant, Michelle, said "My goal (on evening visits) was to go (back to my) home every night without injury." In order to reduce the level of risk, most interview participants emphasized the importance of making home visits accompanied by coworkers or supervisors. The majority of participants said they asked someone to visit clients' homes together in the following situations: 1) evening home visit, 2) meeting with hostile clients, and 3) female worker going into male clients' homes by themselves.

Although accompanied home visits with coworkers or supervisors helped in reducing the level of safety concerns, some participants said that home visiting in pairs may provide a "bipolar advantage" for child welfare workers. For instance, one interview participant said that "two people going into the home can put a client on a defensive." In addition, different perspectives were also found between the supervisors and line workers. Line workers mentioned that supervisors do not want to go to home visits with line workers due to the heavy administrative work duties. However, one supervisor, Jack, mentioned that supervisors or managers sometimes hesitate to accompany line workers because they are concerned that this could undermine child welfare workers' professional competence and clinical skills, and not be perceived as a support:

How will the family perceive me [line worker] if I have to be accompanied by my supervisor on a home visit, will they [clients] think that I'm still in training or I'm still learning how to deliver professional social work services. Because clearly if my supervisor has to come with me, the family will lose some regard or respect for me [line worker] as a professional. I [supervisor] can understand that [line worker's] concern so some of that is how both the worker and the supervisor present the fact that the supervisors are accompanying the worker. (Jack)

\section{Community/Environmental Characteristics}

Visiting communities with a violent reputation, receiving 
little support from police or community members, and a lack of community resources in risky situations posed increased risks during home visits. Melissa noted that she worried about her own safety while traveling in high risk areas in the evening. She added that high-risk communities are sometimes very closed, so community residents saw visitors, including child welfare workers, as a threat to their community. She recalled her past experience of being intimidated by teenagers:

So it was dawn of day but there was a lot of teenagers hanging outside and I had to get to my car and when I was trying to back up to get out of the community, they [teenagers] wouldn't move. They were standing there with me in front of the car; they were standing around the car. They wondered, "What are you doing in our neighborhood?" So they [teenagers] tried to intimidate me and they would just stand there blocking the street.

A lack of community resources emerged as a theme that related to child welfare workers' safety concerns. When workers are concerned about their personal safety while they visit clients' homes, they usually request help from police to ensure their safety. However, some participants felt little respect from police, which could place them at higher risk. For example, Pat said that "sometimes workers had to wait too long to receive help from the police," and some police officers did not know what social workers were expected to do in their communities; therefore, their assistance was not helpful. Similarly, in the court system, there is not enough respect for child welfare workers who are advocating for children and presenting at court hearings.

Another participant, Melissa, said that sometimes she was feeling "stuck out within the community," because she felt that there was a lack of community resources. Amy also discussed that it is vital for child welfare workers to have abundant community resources from which they can seek help, especially when they are exposed to an unsafe working environment:

We [should] know that we have the support out there; from the media, from the police officers, from the community associates. ...I think we should be a little bit more networked, a little bit more tighter on knowing who those community leaders are, knowing who those community advocates are in those communities that we can contact when we need that help. So we need a little bit more network, there are some (resources) out there. (Amy)

\section{Discussions}

The child welfare workers who were interviewed perceived that addressing personal safety is critical to enhance workers' well-being. Consistent with previous research (Spencer and Munch, 2003), the workers acknowledged facing significant risks while conducting home visits. Although the interview participants in the current study had not experienced actual physical attacks by clients during their career, they frequently experienced verbal or physical threats from clients and faced unsafe environmental conditions during their home visits. They sometimes had been victimized by non-clients and had concerns about being a victim while visiting neighborhoods known as drug or gang areas, or where recent incidents of violence had occurred. Also consistent with previous studies (Newhill, 2004; Spencer and Munch, 2003), child welfare workers were concerned about risks to their personal health from visiting clients' homes with sanitation challenges; this is rarely discussed in the literature and practice settings.

As suggested in the Cognitive-Perceptual Model of Risk in Home Visiting (CPMRHV), a child welfare worker's perception of risk is affected by several characteristics, including worker and community characteristics. Individual worker's demographic characteristics, such as age and tenure, were perceived as important factors that increased perception of risk among child welfare workers. The participants believed that a younger worker with less experience may perceive a higher level of risk than an older worker with more tenure. This perception supports earlier studies (Jayaratne, 2004; Ringstad, 2005) in that younger workers are at greater risk, reporting more incidents of physical threat or verbal abuse than older workers. As suggested in prior research (Cohen-Callow et al., 2010), older workers may have developed more coping mechanisms than younger workers to deal with violent situations; therefore, they perceived a lower level of risk while working in communities. However, considering the mixed results in previous studies examining associations between worker characteristics and workplace violence, the interpretation should be cautious and needs further exploration. Given that the most of the interview participants in this study were relatively older with more experiences, they may have biased perspectives regarding the level of risks or clinical skills toward younger child welfare workers. In addition, from this study situational and community factors were also identified with increased risk in home visits. This supports the CMPHRV model and Spencer and Much (2003)'s model and suggests that the situational context of the home visit (i.e., lack of community resources, lack of organizational policies and strategies for personal safety, and the general appearance of the home) contributed to workers' perception of risk. The findings suggested that having enough community resources and receiving adequate support from other professionals help in alleviating child welfare workers' safety concerns.

\section{Study Limitations and Strengths}

This study has several limitations. First, the sample is very small; quantitative survey data were collected in 2007, and follow-up qualitative data were collected in 2011. As the qualitative data were embedded within larger quantitative data with the intent of explaining why certain individual and organizational factors, tested in the quantitative first phase, are significant or not significant predictors of child welfare 
workers' job withdrawal, the potential pool for the qualitative interviews were limited based on scores obtained from the first phase of quantitative data. In addition, about $50 \%$ of potential interview participants had left their organizations since 2007, and the researcher could not obtain their personal contact information for the follow-up qualitative interviews due to privacy restrictions. Possibly, workers who had experienced violent events had already left their agencies at the time of this data collection. Second, despite efforts to rigorously analyze qualitative data, researcher bias and subjectivity may still exist as one researcher conducted all the interviews and analyses. Third, there may have been some respondent concerns about how much information to share in the interviews. Questions included direct or indirect experiences of workplace violence and some participants may have been hesitant to reveal unpleasant truths in order to protect their privacy.

Despite the limitations, the study has several strengths. First, the sample for the qualitative interviews came from a large quantitative study, and therefore, reduces the threats to trustworthiness through triangulation by data source strategy. Second, the use of a member checking strategy enhanced verification of the accuracy and validity of the data as the researcher provided interview summaries and findings to respondents for their feedback. Finally, this study provided a better understanding of child welfare workers' safety experiences and their home visit risks, which contributes to filling the gap left by previous studies.

\section{Implications and Conclusions}

One of the key findings from this study is that external stressors outside of the agencies, including negative public perception and lack of community resources may exacerbate worker safety concerns during home visits. This suggests that child welfare workers, including supervisors and managers, need to actively build and maintain a good reputation by expanding collaborative efforts with community members that includes community resource development, resource mapping, and community relationship building to address worker safety and retention.

Educational opportunities may help in alleviating the perceived risks and improve child welfare workers' safety, especially since the participants in this study reported that they had little or no safety training/education experiences in schools of social work. Safety content needs to be integrated through social work practice courses that includes appropriate assessment of potential violence, interventions to prevent violence from escalating, and development of an emergency management plan and agency policies on safety.

Ultimately, social work agencies must be committed to providing ongoing safety training to both line workers and supervisors/managers to promote a safer work environment and allow workers to share their experiences with other colleagues. Recently, the National Association of Social Workers (NASW) Massachusetts Chapter encouraged social workers, including managers, to participate in skill development education and training related to risk assessment and safety promotion (NASW MA Chapter, 2014). The Chapter suggested that knowledge and skills in risk assessment, safety planning, verbal de-escalation techniques, and non-violent self-defense should be part of safety training and education.

This study also has implications for legislation to promote workplace safety for social workers. NASW members have been committed to improving social worker safety, and more recently they have been involved in many legislative activities. For example, in 2007, to protect social workers and other professionals working with at-risk populations, the Teri Zenner Social Worker Safety Act (H2165) was introduced to establish federal grants to states to provide safety measures, but it still awaits passage. Some states, such as Massachusetts, Kentucky, and West Virginia, recently passed social worker safety legislation (i.e., An Act to Promote the Public Health Through Workplace Safety for Social Workers, H592 \& S1206; The Boni Frederick bill, Kentucky SB59; and Social Worker Safety Bill, West Virginia SB2566) to improve working conditions for social workers. In addition, other states, such as California, New Jersey, and Washington have adopted safety guidelines for social workers to reduce workplace violence. Therefore, the current study may provide evidence for the development of legislation and state guidelines that improve the safety of social workers and other clinicians.

Overall, this study may provide more beneficial information, and contribute to a better understanding and incentive for the further development of safety training in educational curriculums and agencies, and legislation and state guidelines that improve the safety of child welfare workers and other helping professionals.

\section{REFERENCES}

American Federation of State, County and Municipal Employees (2011) Double jeopardy: Caseworkers at risk helping at-risk kids. Available at: http://www.afscme.org/news/publications/workplace -health-and-safety/double-jeopardy-caseworkers-at-risk-helping-at -ris k-kids/ (accessed 31 August 2016)

Astor, R. A., Behre, W. J., Wallace, J. M., \& Fravil, K. A. (1998). School social workers and school violence: Personal safety, training, and violence programs. Social Work, 43(3), 223-232.

Balloch, S., Pahl, J., \& McLean, J. (1998). Working in the social services: Job satisfaction, stress and violence. British Journal of Social Work, 28(3), 329-350.

Boyatzis RE (1998) Transforming qualitative information: Thematic analysis and code development. Thousand Oaks, CA: Sage Publications, Inc. Child Welfare Information Gateway (2014) In-home services in child welfare. Washington, DC: U.S. Department of Health and Human Services, Children's Bureau. Available at:

https://www.childwelfare.gov/pubPDFs/inhome_services.pdf (accessed 31 August 2016) 
Briggs, F., Broadhurst, D., \& Hawkins, R. (2004). Violence, threats and intimidation in the lives of professionals whose work involves children Australian Institute of Criminology. Retrieved from http://criminologyresearchcouncil.gov.au/reports/200102-15.pdf

Cohen-Callow A, Hopkins KM and Kim HJ (2010) Retaining workers approaching retirement: Why child welfare needs to pay attention to the aging workforce. Child Welfare, 88, 209-228.

Doyle J (2007) Child protection and child outcomes: Measuring the effects of foster care. The American Economic Review, 97(5): $1583-1610$.

Duggan A, Caldera D, Rodriguez K, et al. (2007) Impact of a statewide home visiting program to prevent child abuse. Child Abuse \& Neglect, 31(8): 801-827.

Enosh G, Tzafrir SS and Gur A (2013) Client aggression toward social workers and social services in Israel-A Qualitative analysis. Journal of interpersonal violence, 28(6): 1123-1142.

Jayaratne S, Croxton TA and Mattison D (2004) A national survey of violence in the practice of social work. Families in Society, 85(4): 445-453.

Kendra MA and George VD (2001) Defining risk in home visiting. Public Health Nursing, 18(2): 128-137.

Kim, H. \& Hopkins, K. (2015). Child welfare workers' personal safety concerns and organizational commitment: The moderating role of social support. Human Service Organizations Management, Leadership \& Governance (formerly Administration in Social Work), 39 (2), p. 101-115.

Kolko DJ, Hurlburt MS, Zhang J, et al. (2010) Posttraumatic stress symptoms in children and adolescents referred for child welfare investigation a national sample of in-home and out-of-home care. Child maltreatment, 15(1): 48-63.

Koritsas S, Coles J and Boyle M (2010) Workplace violence towards social workers: The Australian experience. British Journal of Social Work, 40(1): 257-271.

National Association of Social Workers (2013) Issue: Strengthen Child Welfare Service Delivery to Enhance Child and Family Well-Being, Available at: https://www.socialworkers.org/advocac y/documents/issue_brief-child_welfare.pdf (accessed 31 August 2016)

National Association of Social Workers (2014) NASW-MA's Workplace Safety Training Program. Available at: http://www.nas wma.org/?page=SafetyTraining (accessed 31 August 2016)

Newhill, C. E. (1996). Prevalence and risk factors for client violence toward social workers. Families in Society: The Journal of Contemporary Human Services, 77(8), 488-495.

Newhill CE (2004) Client violence in social work practice: Prevention, intervention, and research. New York: The Guilford Press.

Ringstad R (2005) Conflict in the workplace: Social workers as victims and perpetrators. Social Work, 50(4): 305-313.

Shields G, \& Kiser J (2003) Violence and aggression directed toward human service workers: An exploratory study. Families in Society, 84(1): 13-20.

Spencer PC and Munch S (2003) Client violence toward social workers: The role of management in community mental health programs. Social Work, 48(4): 532-544.

Sprang G, Craig C and Clark J (2011) Secondary traumatic stress and burnout in child welfare workers: A comparative analysis of occupational distress across professional groups. Child welfare, 90(6): 149-168.

Staerkel FJ and Spieker S (2006) Unstable housing-A significant challenge for home visiting programs: An early head start case example. Journal of Family Social Work, 10(1): 61-76. 